\title{
Usability Evaluation and Improvement of Mission Planner UAV Ground Control System's Interface
}

\author{
Huibin Jin ${ }^{\mathrm{a}, *}$, Yawei Liu ${ }^{\mathrm{b}}$, Xiaomeng Mu${ }^{\mathrm{c}}$, Mingxia $\mathrm{Ma}^{\mathrm{c}}$, and Jing Zhang ${ }^{\mathrm{c}}$ \\ ${ }^{a}$ General Aviation College, Civil Aviation University of China, Tianjin, 300300, China \\ ${ }^{b}$ Civil Aviation College, Zhengzhou University of Aeronautics, Zhengzhou, 450046, China \\ ${ }^{c}$ Flight Technology College, Civil Aviation University of China, Tianjin, 300300, China
}

\begin{abstract}
This paper aims to improve the usability of Mission Planner UAV ground control system's interface through interface improvement. The questionnaire survey and user interview were utilized to evaluate the usability of the UAV ground control system's interface, and the C programming language was used to improve the usability problems. Then, the eye-tracking experiment was designed to compare the difference in usability between the improved system interface and the original system interface, which was used to verify that the improvement to the system interface was effective. The result showed that the usability problems mainly existed in flexibility, minimal user action, minimal memory burden, and user guidance dimension, and the analysis result of multiple indexes such as task completion time, mouse click and fixation points indicate that the usability of the improved system interface was better than the original system.
\end{abstract}

Keywords: UAV; system interface; usability evaluation; interface improvement; eye-tracking

(Submitted on July 19, 2019; Revised on September 26, 2019; Accepted on October 4, 2019)

(C) 2019 Totem Publisher, Inc. All rights reserved.

\section{Introduction}

With the rapid development of the unmanned aerial vehicle (UAV) industry, the number of UAVs is growing exponentially, and their consumers have gradually become public. In recent years, accidents involving UAVs have occurred frequently, and the main problem has been that the accidents are caused by human error. Kim [1] pointed out that this is due to the difference between human and machine. In the field of human-computer interaction, the effective usability of the user interface plays a vital role in promoting human-computer interaction. As the main interactive window between the UAV and user, the usability of the UAV ground control system's interface has a direct impact on the user's operational performance as well as the flight quality of UAV. Therefore, it is critical to improve the usability problems of the UAV ground control system's interface.

Newman [2] and Liu Ying [3] believe that the usability evaluation of design prototypes is an effective way to improve the human-computer interaction interface, which has been widely applied in many fields, including mobile phone interface [4], public facilities interface [5], web interface [6] and other fields [7-9]. Additionally, many scholars believe that scientific evaluation methods and a complete evaluation system will contribute to the examination and improvement of the user interface, so a large amount of research on usability evaluation methods has emerged. After years of development, a variety of evaluation methods have been developed, including subjective evaluation methods such as heuristic evaluation [10] and cognitive walkthrough [11], objective evaluation methods such as eye-tracking technology [12] and an Event-Related Potentials (ERP) experiment [13]. Irizarry [14] used the heuristic evaluation method to discover the usability problems of the UAV control system's interface during a security inspection and put forward corresponding improvement suggestions. However, the single usability evaluation method greatly weakens the reliability of research. Nielsen [13] once made recommendations on the selection of usability evaluation methods, and he believed that the usability study with a combination of multiple evaluation methods can improve the study's reliability. Kim [1] uncovered nine factors and 31 problems affecting the usability of the UAV control system through heuristic evaluation and combined the card 
classification method and user interview to determine the importance of each influencing factor. However, the above research only focuses on the usability problem of the user interface, and does not implement the improvement of the system interface, which greatly reduces the value of usability research.

Therefore, this study used the combination of the questionnaire survey and user interview to evaluate the usability of the Mission Planner UAV ground control system's interface. The C programming language was used to improve the interface's usability problems, and the eye-tracking experiment was designed to verify the effectiveness of this improvement and to create a UAV ground control system interface with superior usability, as well as to further stimulate the interaction between the user and UAV.

\section{Usability Evaluation}

\subsection{Method of Usability Evaluation}

According to Nielsen's introduction of usability evaluation methods and suggestions on their selection in the book 'Usability Engineering' [13], combined with the characteristics of the UAV ground control system's interface, a questionnaire survey and user interview were used in tandem to evaluate the usability of the Mission Planner UAV ground control system's interface in this study.

The questionnaire used the Purdue University Usability Test Checklist [15], which is derived from the basic principles of the human information processing model and can evaluate usability from eight dimensions, including compatibility, consistency, flexibility and so on. There were 100 questions in the whole questionnaire, and formula (1) can be used to obtain the usability score.

$$
F=\frac{\sum w_{i} \times\left(s_{i}-p_{i}\right)}{7 \times \sum\left(w_{i} \times I_{i}\right)} \times 100
$$

Where $F$ is the usability score, $i$ refers to question $i, s_{i}$ means the score obtained on question $i, p_{i}=1$ means question $i$ is applicable but does not exist, and $p_{i}=0$ means question $i$ is not applicable, $I_{i}=1$ means question $i$ is applicable, $I_{i}=0$ means question $i$ is not applicable and $w_{i}$ refers to the importance score of question $i$.

The user interview refers to the research data is obtained through face-to-face conversation with users, which is more flexible and can provide a deeper understanding of users' views and experiences to supplement the questionnaire data. At the same time, this method can make up for the shortcomings of the questionnaire survey method that is prone to misunderstandings.

\subsection{Procedure of Usability Evaluation}

To more effectively and accurately discover the usability problems of the system interface, this survey is mainly aimed at enterprise users who are familiar with the system. The specific usability evaluation procedures are as follows:

- The survey was conducted by using the Purdue University Usability Test Checklist. Before that, respondents were required to follow the following three steps to answer each question:

a. Evaluate the applicability of each question, and if not applicable, skip to the next question. If applicable, please continue to answer the following two questions.

b. Evaluate the importance of the question (1 means the least important, 3 means the most important).

c. Evaluate the performance of the system interface on this question ( 1 means very bad, 7 means very good), if it doesn't exist, choose a non-existent item.

- Questionnaires were collected and screened to obtain the final valid questionnaires.

- User interview was conducted for the selected valid questionnaires' surveyors to further understand the experience process of users and the usability problems they perceived. Interviewees made detailed records. 


\subsection{Data Analysis and Usability Evaluation Results}

Nielsen [16] proposed that there is a relationship between the number of respondents and the number of usability problems, as shown in formula (2):

$$
p=1-(1-\lambda)^{n}
$$

Where $p$ refers to the number of usability problems that can be found, $n$ means the number of respondents, and $\lambda$ refers to the proportion of the number of usability problems found by a respondent to the total number of usability problems.

Additionally, Nielsen found $\lambda \approx 31 \%$ through statistics of past usability experiments, with more and more first-line support for self-design, which has formed the current standard practice [17]. The total number of valid questionnaires collected in this study was 7 , that is $n=7$. According to the calculation of Formula (2), this process can discover about $92.55 \%$ of all usability problems. Therefore, it is considered that the usability evaluation results of 7 respondents are effective.

\subsubsection{Data Analysis of Usability Test Checklist}

According to the data obtained from the Usability Test Checklist, Formula (1) was utilized to calculate the usability score of the Mission Planner UAV ground control system's interface for each dimension. Single-sample t-tests were performed with 100, 90, and 80 as test values, and the results are shown in Table 1:

Table 1. Single sample t-test results of usability score for each dimension

\begin{tabular}{|c|c|c|c|c|c|c|c|c|}
\hline \multirow{2}{*}{ Dimension } & \multirow{2}{*}{$\mathrm{M}$} & \multirow{2}{*}{$\mathrm{S}$} & \multicolumn{2}{|c|}{$V=100$} & \multicolumn{2}{|c|}{$V=90$} & \multicolumn{2}{c|}{$V=80$} \\
\cline { 4 - 9 } & & & $t$ & $p$ & $t$ & $p$ & $t$ \\
\hline Compatibility & 78.68 & 12.31 & -4.584 & 0.004 & -2.435 & 0.051 & -0.285 & 0.785 \\
\hline Consistency & 78.46 & 9.25 & -6.163 & 0.001 & -3.302 & 0.016 & -0.441 & 0.675 \\
\hline Flexibility & 61.34 & 24.17 & -4.233 & 0.005 & -3.138 & 0.020 & -2.043 & 0.087 \\
\hline Learnability & 74.17 & 5.42 & -12.598 & 0.000 & -7.721 & 0.000 & -2.843 & 0.059 \\
\hline Minimal user action & 65.28 & 17.49 & -5.251 & 0.002 & -3.738 & 0.010 & -2.226 & 0.048 \\
\hline $\begin{array}{c}\text { Minimal memory } \\
\text { burden }\end{array}$ & 62.11 & 15.93 & -6.293 & 0.001 & -4.632 & 0.004 & -2.971 & 0.025 \\
\hline Finitude of perception & 66.04 & 15.46 & -5.811 & 0.001 & -4.100 & 0.006 & -2.388 & 0.054 \\
\hline User guidance & 62.81 & 18.79 & -5.236 & 0.002 & -3.828 & 0.009 & -2.420 & 0.042 \\
\hline
\end{tabular}

When the test value is $100(V=100)$, the single-sample t-test results of the usability score for each dimension are all $\mathrm{p}$ $<0.05$, that is, there is a significant difference between the usability score of each dimension. Similarly, when $V=90$, only the usability score of the compatibility dimension is not significantly different from the test value $90(p=0.051>0.05)$. Therefore, it is considered that the interface is optimal in the compatibility dimension. When the test value is 80 , only the three dimensions of minimal user action, minimal memory burden and user guidance are still significantly different from the test value $(p<0.05)$. Additionally, although the usability score of the flexibility dimension possesses no significant difference from the test value, it has the smallest mean value and the largest standard deviation, that is, there are significant differences in the evaluation of the interface's flexibility among various respondents. Therefore, it is believed that the interface's flexibility also has great usability problems.

According to the results of the feedback from the questionnaire, using the Formula (1) to calculate the availability score of the original version of the software, the total score is 54.65. Based on the questionnaire data of the availability questionnaire, the problem of lower scores in each availability dimension will be calculated. The highest score for each question is 21 , the minimum score is 0 (that is, nonexistent), and the passing score for each question is 12.6. After calculation, there are 40 problems of which the average usability score are less than 12.6 , and the usability is poor. Considering the workload and feasibility of version improvement, this study gives priority to improving projects with high importance.

In summary, according to the data analysis of the Usability Test Checklist, it is found that the interface of the Mission Planner UAV ground control system has usability problems in terms of flexibility, minimal user action, minimal memory burden and user guidance. 


\subsubsection{Data Analysis of User Interview}

According to the usability problems found in the data analysis of the Usability Test Checklist, when combined with the details recorded from the user interview, they further clarify the specific problems existing within the interface of the Mission Planner UAV ground control system. See Table 2 for details:

Table 2. Usability problems of the system interface

\begin{tabular}{|c|l|}
\hline Dimension & \multicolumn{1}{c|}{ Usability problems } \\
\hline $\begin{array}{c}\text { Minimal user action; } \\
\text { minimal memory burden }\end{array}$ & $\begin{array}{l}\text { The drop-down menu has too much content, requiring considerable } \\
\text { effort and time to search and select the target. }\end{array}$ \\
\hline $\begin{array}{c}\text { Minimal user action; } \\
\text { minimal memory burden }\end{array}$ & There are too many ineffectual functions for specific task users. \\
\hline $\begin{array}{c}\text { Learnability; } \\
\text { User guidance }\end{array}$ & $\begin{array}{l}\text { The relevant teaching portal is not provided, and more learning time is } \\
\text { required to users. }\end{array}$ \\
\hline User guidance & The interface does not display the relevant guidelines. \\
\hline
\end{tabular}

\section{Improvement of System Interface}

According to the results of the usability evaluation and considering the complexity of the Mission Planner UAV ground control system's code, in a Visual Studio environment, this study only improved the usability problems of the minimal user action and minimal memory burden based on the $\mathrm{C}$ programming language. The specific improvements are shown in Table 3 and the main interface is shown in Figure 1.

\begin{tabular}{|l|l|}
\hline \multicolumn{2}{|c|}{ Table 3. The improvement contents of the system interface } \\
\hline \multirow{2}{*}{ Dimension } & \multicolumn{1}{|c|}{ Improvement contents } \\
\hline \multirow{3}{*}{$\begin{array}{c}\text { Minimal user action; } \\
\text { minimal memory burden }\end{array}$} & $\begin{array}{l}\text { Only "flight plan" and "flight data" are retained in the menu bar at the } \\
\text { top of the interface. }\end{array}$ \\
\cline { 2 - 3 } & $\begin{array}{l}\text { Only "quick display", "instrument" and "data display log" are } \\
\text { maintained in the data display position }\end{array}$ \\
\cline { 2 - 3 } & $\begin{array}{l}\text { Streamline the drop-down menu by simply retaining the necessities to } \\
\text { perform task 1, and adjust the order of the menu contents }\end{array}$ \\
\cline { 2 - 3 } & Keep only three types of map in the map selection \\
\hline
\end{tabular}

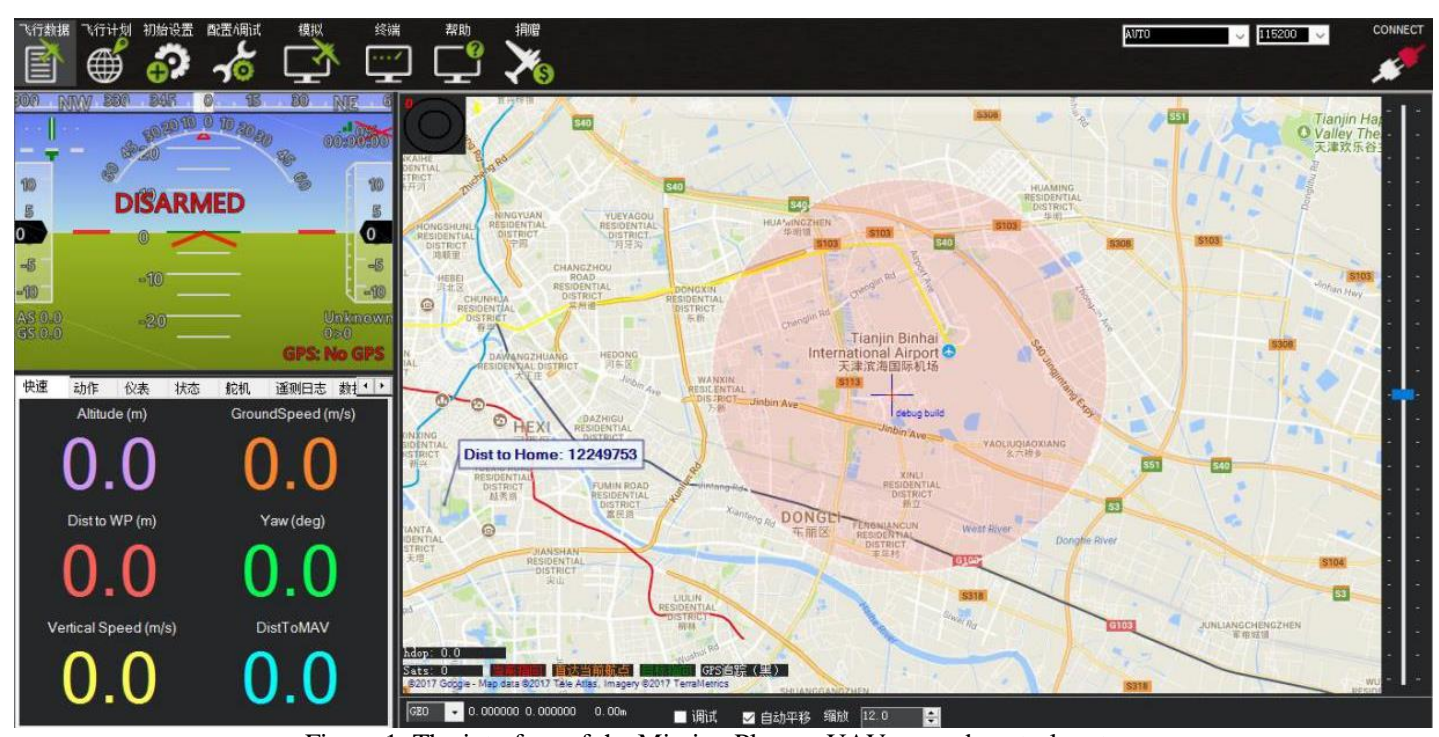

Figure 1. The interface of the Mission Planner UAV ground control system

\section{Usability Comparison Experiment based on Eye-Tracking}

This study designed an eye-tracking experiment to verify whether the usability of the improved system interface has improved. According to the definition of usability in ISO 9241 [18], the difference in usability between the improved system interface and original system interface were compared in terms of effectiveness, efficiency and satisfaction degree. As shown in Table 4. 
Table 4. Test index and content of usability comparison

\begin{tabular}{|c|c|l|}
\hline Usability index & Measurement index & \multicolumn{1}{c|}{ Remark } \\
\hline Effectiveness & Task completion rate & - \\
\hline \multirow{3}{*}{ Efficiency } & Task completion time & $\begin{array}{l}\text { The time taken between the subject entering the } \\
\text { interface and clicking the close button. }\end{array}$ \\
\cline { 2 - 3 } & Average time between mouse clicks & Average time interval between consecutive mouse clicks \\
\cline { 2 - 3 } & $\begin{array}{c}\text { The number of fixation points when } \\
\text { first clicking the target }\end{array}$ & $\begin{array}{l}\text { The number of fixation points formed when the subject } \\
\text { entered the interface and clicked "flight plan". }\end{array}$ \\
\cline { 2 - 3 } $\begin{array}{c}\text { Satisfaction } \\
\text { degree }\end{array}$ & Average fixation time & - \\
\hline
\end{tabular}

\subsection{Subject}

Subjects are 8 graduate students who had not used the experimental system software before, and their eyesight meets the requirements of the eye-tracking experiment.

\subsection{Experiment Platform}

The experimental platform consists of the Mission Planner UAV ground control system software, EyeSo eye tracker and HD video capture device. EyeSo eye tracker is utilized to record the eye-movement data in the experiment, with a sample rate of 60fps and an accuracy of $0.1^{\circ}$.

\subsection{Experiment Task}

According to the above usability problems, combined with the actual operational requirements, a total of three experimental tasks are designed.

\subsubsection{The First Experiment Task}

Drawing the flight range and refining the flight path of the UAV further, then selecting a certain height and configuring the camera, finally taking pictures of the flight path's ground condition by the UAV. The steps are as follows: enter the software -flight plan -select the Bing mixed map - (right-click at any point on the map) to draw the polygon - add polygon points (add four points) (right-click on the map) automatic waypoint -Survey (Grid)-Select any camera you are familiar with (if you are not familiar with the camera rule, Optional)-Altitude(100)-Accept-End (close the dialog, close the software) -End the task .Detailed task flow is shown in Figure 2.

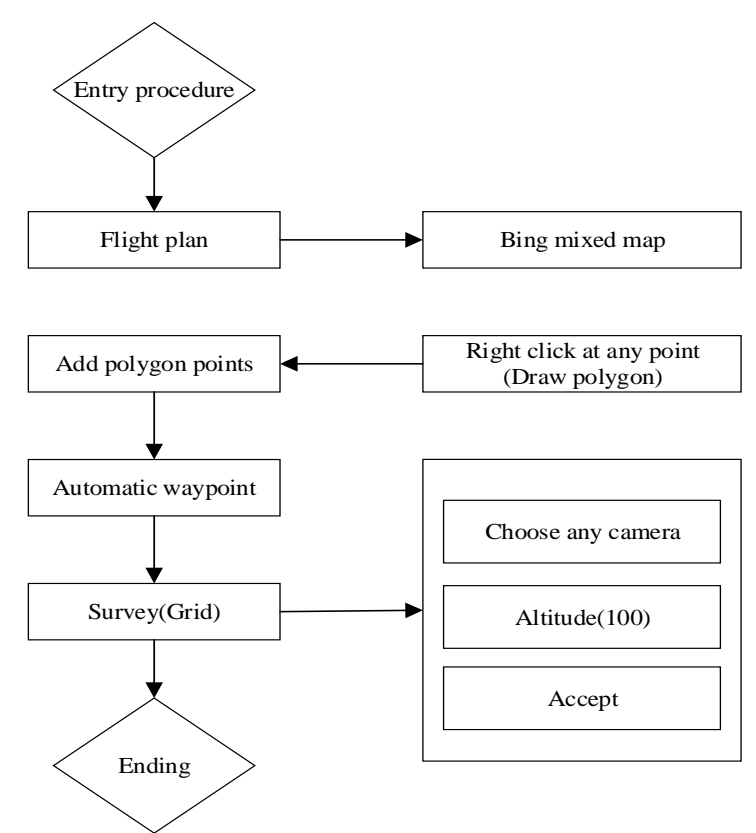

Figure 2. The flow chart of first task 


\subsubsection{The Second Experiment Task}

When the UAV is flying, measuring the distance between any two points on the map through the UAV ground control system. The steps are as follows: enter the software - flight plan - select the Bing mixed map - (right-click on the map) map tool - measure the distance (appear a point) - another point on the map, left-click then right click - Map tool - measure distance - (after the dialog box appears) click "OK" - close the software - the task ends. Detailed task flow is shown in Figure 3 .

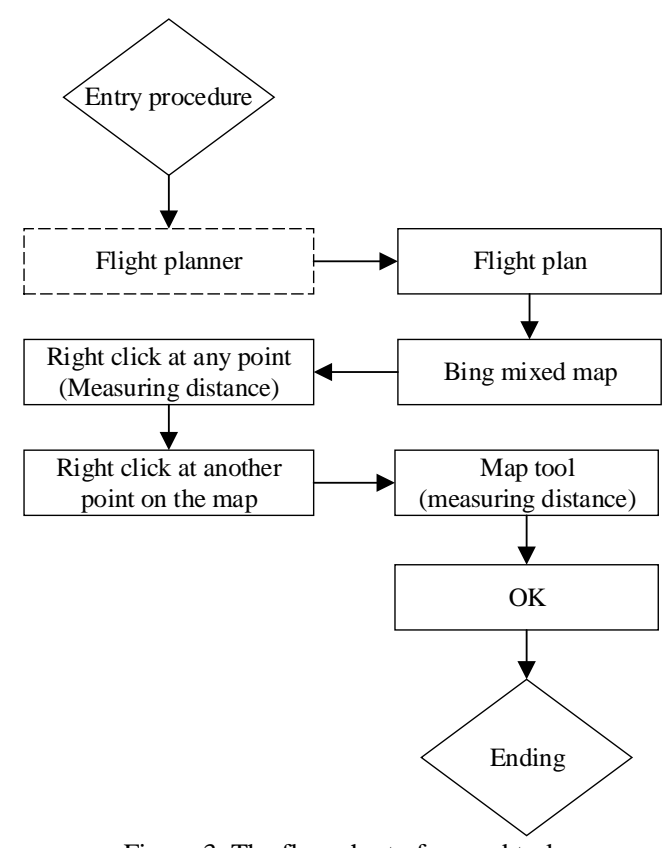

Figure 3. The flow chart of second task

\subsubsection{Experiment Task Two}

Importing the pre-set file of the UAV flight path into the UAV ground control system. The steps are as follows: enter the software - flight plan - load the way-point file (select the file named "way-point file" on the desktop) - close the software end of the task. If you open the file, there is only one interface, right-click on the map location, select "Flight Planner" - load the way-point file (select the file named "way-point file" on the desktop) - enlarge the map, and move the location of the point to the center of the software interface. Detailed experimental process is shown in Figure 4.

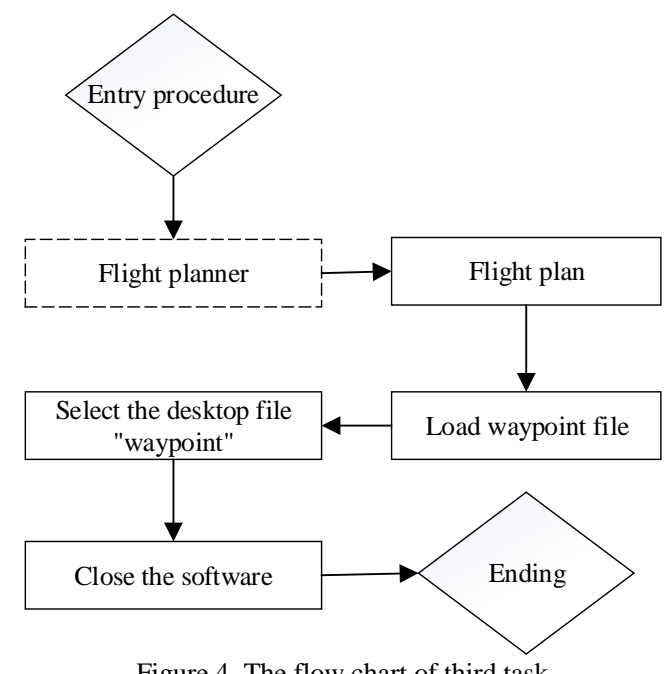

Figure 4. The flow chart of third task

Each subject was required to complete the above tasks in both the original system and the improved system. 


\subsection{Experiment Procedure}

Before the experiment, the basic operation of the experiment task and the experiment procedure were explained to the subjects. After the subjects had a certain understanding of the experiment, the experiment began. The experiment procedure can be divided into the following four steps:

- To avoid the increase in subjects' familiarity with the experiment task, which was caused by the sequential experiment, before the experiment, the Latin square experiment sequence was designed for the subjects, and each task is performed in this order.

- The experimenter debugs the HD video capture device to record the experiment process. Then they set the relevant parameters of the eye tracker and help the subject to calibrate the eye tracker.

- Each time the task is completed, the experimenter saves the relevant experiment data to the specified file.

- After each subject completes the experiment, the experimenter disconnects the device and the task is completed.

\subsection{Experiment Data Analysis}

\subsubsection{Data Screening}

Data screening should be carried out first to ensure the effectiveness of data analysis. The experiment requires that the subjects' data sampling rate be more than $80 \%$. The result of data statistics showed that one subject's data did not meet the requirements. Therefore, the data of this subject will be removed and further analysis will not be carried out.

\subsubsection{Data Analysis}

a. Task completion rate

According to the basic operational requirements of the experiment task, all subjects have completed tasks in both the original system and the improved system, the completion rate of the task reached $100 \%$. Therefore, it is considered that there is no difference in the effectiveness between the original system's interface and the improved system's interface.

\section{b. Task completion time}

Firstly, the Romanov criterion (t-test criterion) is tested, and the gross error data of the experimental task total time data obtained in different versions of each task is tested. The result shows that there is no gross error. Further, the time taken by subjects to complete the experiment task with the original system and improved system are shown in Figure 5. The Krelated sample test was used to analyze the difference in data. The results showed that the task completion time in the improved system is significantly lower than that in the original system $(p<0.05)$, which indicates that the improvement of the interface can improve the user's operational efficiency.

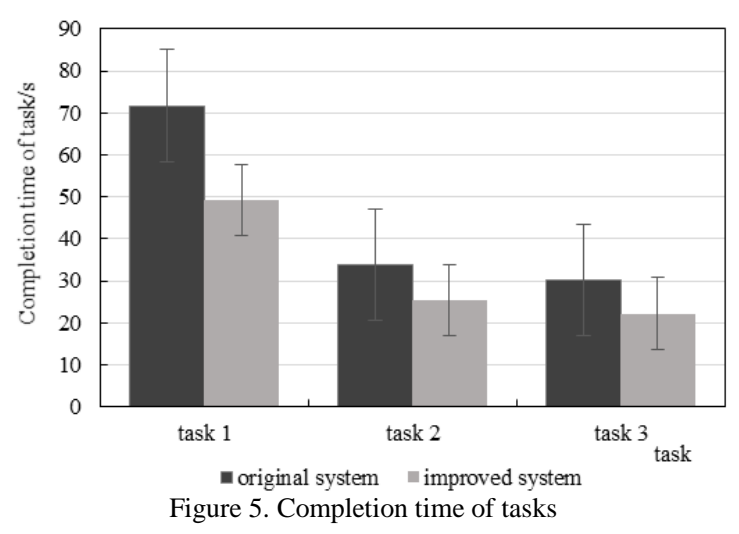

c. Average time between mouse clicks

The length of the mouse click interval is also used in the usability evaluation of the software. When the user has not operated the mouse for a long time, it can be considered that the user is reading the data in the software interface. In other words, the longer the interval between mouse clicks, the longer the process of reading messages in the software interface, 
the easier the content in the software interface is confused by the subject. The greater the cognitive load of users, the worse the usability of the software. According to the statistics, in each stage of the different experiment tasks, the average time between mouse clicks in the improved system is lower than that in the original system. The difference among data was analyzed through the K-related sample test, and the results are shown in Table 5. Among all the experimental tasks, only the task execution phase of task 1 has no significant difference. If the interference of the learning effect is excluded, it can still be considered that the target search efficiency of subjects in the improved system interface is higher.

Table 5. Data analysis results of average mouse click interval time (unit: $\mathrm{ms}$ )

\begin{tabular}{|c|c|c|c|c|c|}
\hline Task stage & Task & Original system & Improved system & $\chi^{2}$ & $p$ \\
\hline \multirow{3}{*}{ Software open } & 1 & 514 & 480 & 6.532 & 0.041 \\
\cline { 2 - 6 } & 2 & 567 & 492 & 8.657 & 0.034 \\
\cline { 2 - 6 } & 3 & 524 & 548 & 7.114 & 0.048 \\
\hline \multirow{3}{*}{ Task execution } & 1 & 791 & 742 & 9.000 & 0.11 \\
\cline { 2 - 6 } & 2 & 862 & 687 & 5.429 & 0.046 \\
\cline { 2 - 6 } & 3 & 815 & 679 & 7.286 & 0.050 \\
\hline
\end{tabular}

d. The number of fixation points when first clicking the target

The comparative analysis and the significant difference analysis of the number of fixation points when subjects clicked the target at first are shown in Figure 6, the results show that in tasks 1 and 2, the average number of fixation points when subjects clicked the target at first in the improved system is significantly lower than that in the original system $(p<0.05)$, and there is no significant difference in task 3 , which may be due to the learning effect.

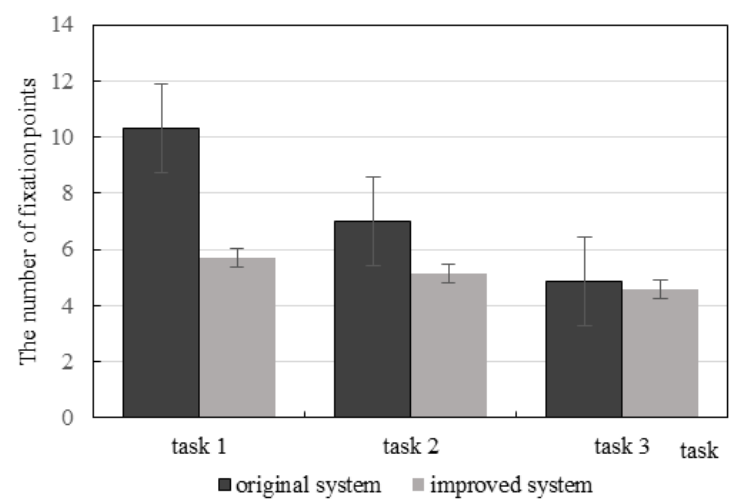

Figure 6. The average number of fixation points when first clicking target

\section{e. Average fixation time}

The duration of gaze is an important indicator to reflect usability. Generally speaking, the longer the gaze duration, the more perplexed to detect the area of the software, that is, the worse usability of the software is. The K-related sample test was used to analyze the difference in the average fixation time between the original system interface and the improved system interface at each stage of the experiment tasks. The results are shown in Table 6 . The average fixation time in the improved system interface is significantly lower than that in the original system interface. Therefore, it was assumed that the interface improvement can improve the user's understanding of the interface's content and reduce the cognitive processing time.

Table 6. K-related sample test results of average fixation time

\begin{tabular}{|c|c|c|c|c|c|}
\hline Task stage & Task & Original system & Improved system & $\chi^{2}$ & $p$ \\
\hline \multirow{3}{*}{ Software open } & 1 & 506 & 471 & 6.333 & 0.042 \\
\cline { 2 - 6 } & 2 & 553 & 518 & 7.971 & 0.047 \\
\cline { 2 - 6 } & 3 & 455 & 458 & 6.429 & 0.039 \\
\hline \multirow{3}{*}{ Task execution } & 1 & 586 & 559 & 7.524 & 0.035 \\
\cline { 2 - 6 } & 2 & 553 & 568 & 5.429 & 0.046 \\
\cline { 2 - 6 } & 3 & 475 & 452 & 7.286 & 0.043 \\
\hline
\end{tabular}

\section{f. Satisfaction degree}

When subjects completed the experiment, they all expressed that they were more satisfied with the operation on the improved system interface. Therefore, the user's satisfaction degree with the improved system interface is considered to be 
better than that with the original system interface.

In summary, there is no difference in effectiveness between the improved system interface and the original system interface, however, in terms of efficiency and satisfaction degree, the improved system interface is better than the original system interface.

\section{Conclusions}

The usability evaluation of the Mission Planner UAV ground control system's interface was carried out through a combination of a questionnaire survey and user interview. It was found that the usability problems primarily existed in flexibility, minimal user action, minimal memory burden and user guidance dimension. Also, the usability problems have been improved through the $\mathrm{C}$ programming language. Usability evaluation is an effective way to improve the user interface.

Through the eye-tracking experiment, it was verified that the usability of the improved system interface has been improved. Eye-tracking technology can objectively evaluate the usability of the user interface.

The system interface with better usability obtained in this paper will be better able to realize the interaction between users and UAVs, which is of great significance for improving the user's operational performance and ensuring the flight safety of the UAVs further.

\section{Acknowledgements}

The authors were supported by the National Key Research and Development Program: Wide Area Aviation Safety Surveillance Technology and Application (No.2016YFB0502400), Fundamental Research Funds for the Central Universities (No.3122017014).

\section{References}

1. M. C. Cha, B. M. Kim, J. I. Lee, and Y. G. Ji, "Usability Evaluation for User Interface of a Drone Remote Controller," in Proceedings of the 2016 Autumn Meeting of the Korean Society of Ergonomics, pp. 417-424, 2016

2. W. M. Newman, M. G. Lamming, and M. Lamming, "Interactive System Design," Interactive System Design, 1995

3. Y. Liu, "Usability Evaluation and Method of Human-Computer Interaction Interface," Chinese Journal of Ergonomics, Vol. 8, No. 2, pp. 35-38, 2002

4. Y. G. Ji, J. H. Park, C. Lee, and M. H. Yun, "A Usability Checklist for the Usability Evaluation of Mobile Phone User Interface," International Journal of Human-Computer Interaction, Vol. 20, No. 3, pp. 207-231, 2006

5. M. Shafiq, M. Ahmad, and J. G. Choi, "Public System Usability Analysis for the Cognitive Burden and Interface Standardization: A Case Study of Cross-ATM Design," Journal of Organizational Computing and Electronic Commerce, Vol. 27, No. 2, pp. 162-196, 2017

6. H. B. Jin, Y. W. Liu, and X. M. Mu, "Evaluation of the Usability of Airline Official Website based on Eye Tracking," Packaging Engineering, Vol. 39, No. 10, pp. 173-176, 2018

7. Y. Tu, Y. Lin, J. Wang, and J. -U. Kim, "Semi-Supervised Learning with Generative Adversarial Networks on Digital Signal Modulation Classification," CMC-Computers Materials and Continua, Vol. 55, No. 2, pp. 243-254, 2018

8. Y. Lin, X. Zhu, Z. Zheng, Z. Dou, and R. Zhou, "The Individual Identification Method of Wireless Device based on Dimensionality Reduction and Machine Learning," Journal of Supercomputing, No. 5, pp. 1-18, 2017

9. Z. Y. Zhang, X. H. Guo, and Y Lin, "Trust Management Method of D2D Communication based on RF Fingerprint Identification," IEEE Access, No. 6, pp. 66082-66087, 2018

10. J. Nielsen, "Usability Engineering," China Machine Press, 2004

11. L. Clayton and R. John, "Task-Centered User Interface Design: A Practical Introduction," Colorado University of Colorado, 1994

12. Q. Liu, C. Q. Xue, and F. Hoehn, "Interface Usability Evaluation based on Eye-Tracking Technology," Journal of Southeast University (Natural Science Edition), Vol. 40, No. 2, pp. 331-334, 2010

13. J. Y. Shen, C. Q. Xue, and Y. F. Niu, "Design Methods Research of Human-Computer Interface based on Event-Related Potential," Design, No. 6, pp. 134-135, 2018

14. J. Irizarry, M. Gheisari, and B. N. Walker, "Usability Assessment of Drone Technology as Safety Inspection Tools," Electronic Journal of Information Technology in Construction, Vol. 17, pp. 194-212, 2012

15. J. M. Dong, L. M. Fu, and G. Salvendy, "Human-Computer Interaction: User-Centered Design and Evaluation," Tsinghua University Press, 2010

16. J. Nielsen, "Usability laboratories," Behaviour and Information Technology, Vol. 13, No. 1, pp. 3-8, 1994

17. T. Tetsuya, "User Experience and Usability Testing," Post and Telecom Press, 2015

18. ISO 9241-11, "Ergonomic Requirements for Office Work with Visual Display Terminals, Part1 1: Guidance on usability," 1998 